\title{
Multiwavelength observations of a VHE gamma-ray flare from PKS 1510-089 in 2015
}

\author{
M. L. Ahnen ${ }^{1}$, S. Ansoldi ${ }^{2,25}$, L. A. Antonelli ${ }^{3}$, C. Arcaro ${ }^{4}$, A. Babić ${ }^{5}$, B. Banerjee ${ }^{6}$, P. Bangale ${ }^{7}$,
} U. Barres de Almeida ${ }^{7,26}$, J. A. Barrio ${ }^{8}$, W. Bednarek ${ }^{11}$, E. Bernardini ${ }^{12,28}$, A. Berti²,29, B. Biasuzzi ${ }^{2}$, A. Biland ${ }^{1}$, O. Blanch ${ }^{13}$, S. Bonnefoy ${ }^{8}$, G. Bonnoli ${ }^{14}$, F. Borracci ${ }^{7}$, T. Bretz ${ }^{15,30}$, R. Carosi ${ }^{14}$, A. Carosi ${ }^{3}$, A. Chatterjee ${ }^{6}$, P. Colin $^{7}$, E. Colombo ${ }^{9,10}$, J. L. Contreras ${ }^{8}$, J. Cortina ${ }^{13}$, S. Covino ${ }^{3}$, P. Cumani ${ }^{13}$, P. Da Vela ${ }^{14}$, F. Dazzi ${ }^{7}$, A. De Angelis ${ }^{4}$, B. De Lotto ${ }^{2}$, E. de Oña Wilhelmi ${ }^{16}$, F. Di Pierro ${ }^{3}$, M. Doert ${ }^{17}$, A. Domínguez ${ }^{8}$, D. Dominis Prester ${ }^{5}$, D. Dorner ${ }^{15}$, M. Doro ${ }^{4}$, S. Einecke ${ }^{17}$, D. Eisenacher Glawion ${ }^{15}$, D. Elsaesser ${ }^{17}$, M. Engelkemeier ${ }^{17}$, V. Fallah Ramazani ${ }^{18}$, A. Fernández-Barral ${ }^{13}$, D. Fidalgo ${ }^{8}$, M. V. Fonseca ${ }^{8}$, L. Font ${ }^{19}$, C. Fruck ${ }^{7}$, D. Galindo ${ }^{20}$, R. J. García López ${ }^{9,10}$, M. Garczarczyk ${ }^{12}$, M. Gaug ${ }^{19}$, P. Giammaria ${ }^{3}$, N. Godinović ${ }^{5}$, D. Gora ${ }^{12}$, D. Guberman ${ }^{13}$, D. Hadasch ${ }^{21}$, A. Hahn ${ }^{7}$,

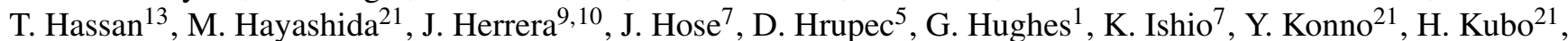

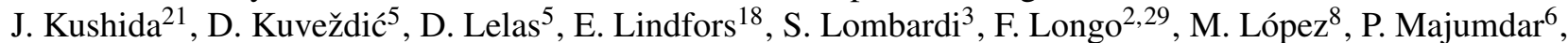
M. Makariev ${ }^{22}$, G. Maneva ${ }^{22}$, M. Manganaro ${ }^{9,10}$, K. Mannheim ${ }^{15}$, L. Maraschi ${ }^{3}$, M. Mariotti $^{4}$, M. Martínez ${ }^{13}$, D. Mazin ${ }^{7,31}$, U. Menzel ${ }^{7}$, R. Mirzoyan ${ }^{7}$, A. Moralejo ${ }^{13}$, E. Moretti ${ }^{7}$, D. Nakajima ${ }^{21}$, V. Neustroev ${ }^{18}$,

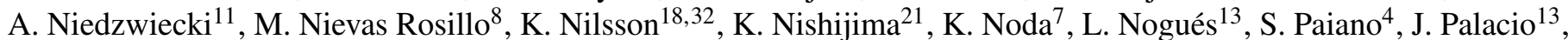
M. Palatiello ${ }^{2}$, D. Paneque ${ }^{7}$, R. Paoletti ${ }^{14}$, J. M. Paredes ${ }^{20}$, X. Paredes-Fortuny ${ }^{20}$, G. Pedaletti ${ }^{12}$, M. Peresano ${ }^{2}$, L. Perri ${ }^{3}$, M. Persic ${ }^{2,33}$, J. Poutanen ${ }^{18}$, P. G. Prada Moronii ${ }^{23}$, E. Prandini ${ }^{4}$, I. Puljak ${ }^{5}$, J. R. Garcia ${ }^{7}$, I. Reichardt ${ }^{4}$, W. Rhode ${ }^{17}$, M. Ribó ${ }^{20}$, J. Rico ${ }^{13}$, T. Saito ${ }^{21}$, K. Satalecka ${ }^{12}$, S. Schroeder ${ }^{17}$, T. Schweizer ${ }^{7}$, S. N. Shore ${ }^{23}$, A. Sillanpää ${ }^{18}$, J. Sitarek ${ }^{11, \star}$, I. Šnidarićc ${ }^{5}$, D. Sobczynska ${ }^{11}$, A. Stamerra ${ }^{3}$, M. Strzys ${ }^{7}$, T. Surić ${ }^{5}$, L. Takalo ${ }^{18}$,

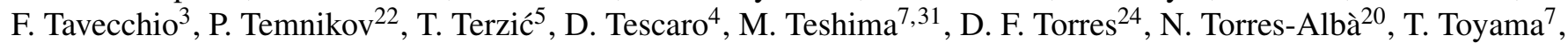

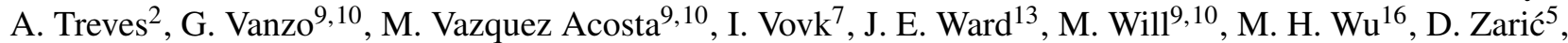
R. Desiante ${ }^{2}$ (MAGIC Collaboration), J. Becerra González ${ }^{9,10,27}$, F. D’Ammando ${ }^{34,35}$,

S. Larsson ${ }^{36,37}$ (Fermi-LAT Collaboration), C. M. Raiteri ${ }^{38}$, R. Reinthal ${ }^{39}$, A. Lähteenmäki ${ }^{40,41}$, E. Järvelä ${ }^{40,41}$ M. Tornikoski ${ }^{40}$, V. Ramakrishnan ${ }^{40}$, S. G. Jorstad ${ }^{42,43}$, A. P. Marscher ${ }^{42}$, V. Bala ${ }^{42}$, N. R. MacDonald ${ }^{42}$, N. Kaur ${ }^{44,45}$, Sameer $^{44,46}$, K. Baliyan ${ }^{44}$, J. A. Acosta-Pulido ${ }^{9,10}$, C. Lazaro, ${ }^{9,10}$, C. Martí-nez-Lombilla ${ }^{9,10}$, A. B. Grinon-Marin ${ }^{9,10}$,

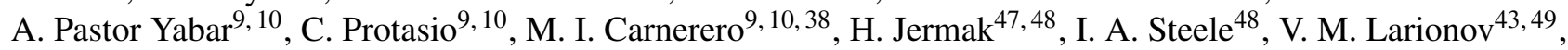
G. A. Borman ${ }^{50}$, and T. S. Grishina ${ }^{43}$

(Affiliations can be found after the references)

Received 25 October 2016 / Accepted 27 February 2017

\begin{abstract}
Context. PKS 1510-089 is one of only a few flat spectrum radio quasars detected in the very-high-energy (VHE, $>100 \mathrm{GeV}$ ) gamma-ray band. Aims. We study the broadband spectral and temporal properties of the PKS 1510-089 emission during a high gamma-ray state.

Methods. We performed VHE gamma-ray observations of PKS 1510-089 with the Major Atmospheric Gamma Imaging Cherenkov (MAGIC) telescopes during a long, high gamma-ray state in May 2015. In order to perform broadband modeling of the source, we have also gathered contemporaneous multiwavelength data in radio, IR, optical photometry and polarization, UV, X-ray, and GeV gamma-ray ranges. We construct a broadband spectral energy distribution (SED) in two periods, selected according to VHE gamma-ray state.

Results. PKS 1510-089 was detected by MAGIC during a few day-long observations performed in the middle of a long, high optical and gammaray state, showing for the first time a significant VHE gamma-ray variability. Similarly to the optical and gamma-ray high state of the source detected in 2012, it was accompanied by a rotation of the optical polarization angle and the emission of a new jet component observed in radio. However, owing to large uncertainty on the knot separation time, the association with the VHE gamma-ray emission cannot be firmly established. The spectral shape in the VHE band during the flare is similar to those obtained during previous measurements of the source. The observed flux variability sets constraints for the first time on the size of the region from which VHE gamma rays are emitted. We model the broadband SED in the framework of the external Compton scenario and discuss the possible emission site in view of multiwavelength data and alternative emission models.
\end{abstract}

Key words. galaxies: active - galaxies: jets - gamma rays: galaxies - quasars: individual: PKS 1510-089

* Corresponding authors: J. Sitarek, e-mail: jsitarek@uni .lodz.pl; J. Becerra González, e-mail: becerragonzalez@gmail.com; E. Lindfors, e-mail: elilin@utu.fi 


\section{Introduction}

PKS 1510-089 is a bright flat spectrum radio quasar (FSRQ) located at a redshift of $z=0.36$ (Tanner et al. 1996). The source is one of only six objects firmly classified as a FSRQ from which gamma-ray emission has been detected in the very-high-energy (VHE, $>100 \mathrm{GeV}$ ) range (Abramowski et al. 2013). Moreover, one of the highest recorded apparent speeds of superluminal motion, up to $\sim 46 c$, has been seen in the ultrarelativistic jet of PKS 1510-089 (Jorstad et al. 2005). As it is in many other FSRQs, the GeV gamma-ray emission of PKS 1510-089 is strongly variable (Abdo et al. 2010; Saito et al. 2013; Aleksić et al. 2014). The doubling time of the PKS 1510-089 flares observed in the GeV range go down to $1 \mathrm{~h}$ (Saito et al. 2013).

Most of the FSRQs have been detected in the VHE gamma-ray range during (usually short) flares (see, e.g., MAGIC Collaboration et al. 2008; Aleksić et al. 2011; Ahnen et al. 2015). Since 2013, the Major Atmospheric Gamma Imaging Cherenkov (MAGIC) telescope system has performed regular monitoring of PKS 1510-089. Interestingly, until 2015, no variability was seen in PKS 1510-089 in VHE gamma rays in H.E.S.S. (Abramowski et al. 2013) or in MAGIC (Aleksić et al. 2014) observations. It should be noted, however, that both VHE gamma-ray detections happened during long periods of enhanced optical and $\mathrm{GeV}$ gamma-ray activity. Hence no low-state VHE gamma-ray emission has been established so far from PKS 1510-089.

In May 2015, a strong flare of PKS 1510-089 was observed in $\mathrm{GeV}$ gamma rays by the Large Area Telescope (LAT) on board the Fermi satellite. At this time the source also showed high activity in the optical (Jankowsky et al. 2015; Mirzoyan 2015) and IR bands (Sameer et al. 2015; Carrasco et al. 2015). The high state triggered further MAGIC observations, which led to the detection of an enhanced VHE gamma-ray activity from the source (Mirzoyan 2015). The VHE gamma-ray emission has been also observed by the H.E.S.S. telescope (Zacharias et al. 2016). In May 2016 another flare occurred (de Naurois 2016; Mirzoyan 2016) with an even stronger VHE gamma-ray flux than in May 2015. The May 2016 flare will be discussed in a separate paper.

In this paper we report on the observations of PKS 1510-089 during the May 2015 flare. In Sect. 2 we briefly introduce the instruments that provided multiwavelength data and describe the data reduction procedures. In Sect. 3 we present the multiwavelength behavior of the source. Section 4 is devoted to the interpretation of the data in the framework of an external Compton model. The most important results are summarized in Sect. 5.

\section{Instruments, observations and data analysis}

During the May 2015 outburst PKS 1510-089 was observed by various instruments in a broad range of frequencies (from radio up to VHE gamma rays). In this section we introduce the different instruments and data sets and explain the data analysis procedure.

\subsection{MAGIC}

MAGIC is a system of two imaging atmospheric Cherenkov telescopes with a mirror dish diameter of $17 \mathrm{~m}$ each. They are located in the Canary Islands, on La Palma $\left(28.7^{\circ} \mathrm{N}, 17.9^{\circ} \mathrm{W}\right)$, at a height of $2200 \mathrm{~m}$ above sea level (Aleksić et al. 2016a). As PKS 1510-089 is a southern source, only observable at zenith angle $>38^{\circ}$, the corresponding trigger threshold is $\gtrsim 90 \mathrm{GeV}$
(Aleksić et al. 2016b), about 1.7 times larger than for the low zenith observations.

The MAGIC telescopes observed PKS 1510-089 for $5.4 \mathrm{~h}$ between 18 and 24 of May, 2015 (MJD 57 160-57 166). The data were analyzed using MARS, the standard analysis package of MAGIC (Zanin et al. 2013; Aleksić et al. 2016b). As part of the data set was affected by Calima ${ }^{1}$ we applied a correction for the atmosphere transmission based on LIDAR information (Fruck \& Gaug 2015).

\subsection{Fermi-LAT}

Fermi-LAT monitors the gamma-ray sky every $3 \mathrm{~h}$ in the energy range from $20 \mathrm{MeV}$ to beyond $300 \mathrm{GeV}$ (Atwood et al. 2009). An analysis of the publicly available Pass 8 SOURCE class events was performed for a region of interest (ROI) of $10^{\circ}$ radius centered at the position of PKS $1510-089$. In order to reduce contamination from the Earth's limb, a zenith angle cut of $<90^{\circ}$ was applied. The analysis was performed with the ScienceTools software package version v10r0p5 using the P8R2_SOURCE_V6 ${ }^{2}$ instrument response function and the $g 11$ _iem_v06 and iso_P8R2_SOURCE_V6_v06 models ${ }^{3}$ for the Galactic and isotropic diffuse emission (Acero et al. 2016), respectively.

An unbinned likelihood analysis was applied using gtlike, including in the model all 3FGL sources (Acero et al. 2015) within $20^{\circ}$ from PKS 1510-089. The spectral indices and fluxes were left free for sources within $10^{\circ}$, while sources from $10^{\circ}$ to $20^{\circ}$ have their parameters fixed to their catalog value. A first unbinned likelihood fit was performed for the events collected within almost four months of data from 22 March 2015 to 19 July 2015 (MJD 57 103-57223) in the energy range between $100 \mathrm{MeV}$ and $800 \mathrm{GeV}$. The sources with a test statistic (TS; Mattox et al. 1996) below 5 were removed from the model. Next, the optimized output model was used to produce the light curves and spectra of PKS 1510-089 in different time bins (from 1 day to $3 \mathrm{~h}$ ) and energy ranges $(E>100 \mathrm{MeV}, E>1 \mathrm{GeV})$. For the calculation of the light curves, all sources were fixed in the model except PKS 1510-089 for which both the flux normalization and the spectral index were left free and modeled as a power law. For the calculation of the spectral points, the spectral index of PKS 1510-089 was also fixed to its best-fit value during the considered time period for which the spectral points are estimated. The normalization of the Galactic and isotropic diffuse emission models was left to vary freely during the calculation of both the light curves and the spectra.

\subsection{Swift-XRT and UVOT}

The multi-epochs (16 individual pointings) event list obtained by the X-ray Telescope (XRT) (Burrows et al. 2004) on board the Swift satellite during the period from 11 May to 25 May 2015 (MJD 57 153-57 167) with the total exposure time of $26.6 \mathrm{ks}$ were downloaded from the publicly available database table SWIFTXRLOG (Swift-XRT Instrument Log). The individual exposures ranged from 0.6 to $4 \mathrm{ks}$. They were processed using the HEASOFT package version 6.18. All the observations from

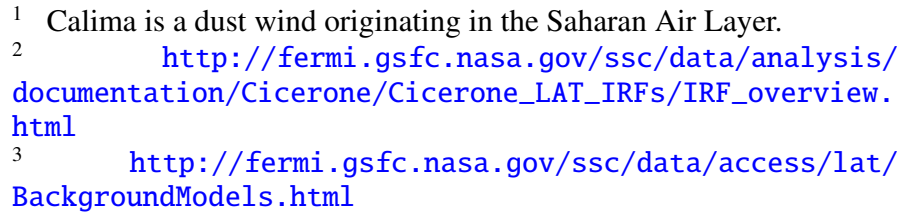


this period were performed in photon counting (PC) mode. The source region was defined as a circle of 20 pixel $\left(\sim 47^{\prime \prime}\right)$ radius at the center of the source, while the background region was defined by an annulus centered at the source with inner and outer radii of $40\left(\sim 94^{\prime \prime}\right)$ and 80 pixels $\left(\sim 188^{\prime \prime}\right)$, respectively. The source and background spectra were extracted using XSELECT task (v2.4c). The source spectrum count rate did not exceed 0.5 counts/s in any of the observation epochs. Therefore, no pile-up correction was needed. For the light curve analysis we combined three pairs of epochs (MJD 57 157, 57 161, 57 163) separated by $\sim 2 \mathrm{~h}$.

The xrtexpomap task (v0.2.7) was used to correct the flux loss caused because some of the CCD pixels were not used during the data collection. The xrtmkarf task (v0.6.3) took into account vignetting and bad pixels. The grppha task was used to group source spectra in such a way that each bin contains 20 counts. XSPEC task (v12.9.0i) was used to calculate the flux and power-law model spectral parameters using a fixed equivalent Galactic hydrogen column density of $n_{\mathrm{H}}=6.89 \times$ $10^{20}\left[\mathrm{~cm}^{-2}\right]$ (Kalberla et al. 2005).

The second instrument on board the Swift satellite, the Ultraviolet/Optical Telescope (UVOT, Poole et al. 2008) was used to monitor the flux of the source in the $180-600 \mathrm{~nm}$ wavelength range. Following Raiteri et al. (2010) we used an iterative procedure for the data calibration, where the effective wavelength, counts-to-flux conversion factor, and Galactic extinction for each filter were calculated by taking into account the filter's effective area and the source's spectral shape. Of the 16 pointings in the investigated time period, 8 were taken with a full set of filters $(v$, $b, u, w 1, m 2, w 2)$. For the SED modeling we used pointings on MJD 57160 (with $u$ and $w 2$ filters) contemporaneous with the MAGIC flaring state, and on MJD 57165 (all filters available) during the post-flare MAGIC observations.

\subsection{Optical photometry and polarization}

PKS 1510-089 is regularly monitored as part of the Tuorla blazar monitoring program ${ }^{4}$ in the $R$ band using a $35 \mathrm{~cm}$ Celestron telescope attached to the KVA (Kunglinga Vetenskapsakademi) telescope located at La Palma. The data analysis was performed with the semi-automatic pipeline using the standard analysis procedures (Nilsson et al., in prep.). The differential photometry was performed using the comparison star magnitudes from Villata et al. (1997). The magnitudes were corrected for the Galactic extinction using values from Schlafly \& Finkbeiner (2011).

The optical polarization observations were performed with a number of instruments: Nordic Optical Telescope (NOT), Steward Observatory, Perkins Telescopes, RINGO3, AZT-8, and LX-200. The NOT polarimetric observations were done with ALFOSC in the $R$ band using the standard setup for linear polarization observations (lambda/2 retarder followed by a calcite). The data were analyzed using the standard procedures with semi-automatic software as in Hovatta et al. (2016). The Steward polarimetric observations were obtained as part of an ongoing monitoring program of gamma-ray-bright blazars in support of the Fermi mission ${ }^{5}$. The observations were performed in the 5000-7000 $\AA$ band. The data analysis pipeline is described in Smith et al. (2009). Polarimetric $R$-band observations were also provided by the $1.8 \mathrm{~m}$ Perkins telescope at Lowell Observatory equipped with PRISM (Perkins Reimaging System).

\footnotetext{
http://users.utu.fi/kani/1m

http://james.as.arizona.edu/ psmith/Fermi
}

The data analysis was done following the standard procedures as in Chatterjee et al. (2008). Polarization observations were also taken with the RINGO3 polarimeter (Arnold et al. 2012) on the fully robotic and autonomous Liverpool Telescope on La Palma, Canary Islands (Steele et al. 2004), as part of the Liverpool blazar monitoring campaign (see Jermak et al. 2016), in collaboration with the Monitoring AGN Polarimetry at the LIverpool Telescope (MAPLIT) program. Simultaneous observations in the blue, $350-640 \mathrm{~nm}$; green, 650-760 nm; and red, 770-1000 nm, passbands were taken using a rapidly rotating (once per $4 \mathrm{~s}$ ) polaroid which modulates the incoming beam of light in eight rotor positions. For this work we use only the 650-760 nm measurements, which are the closest to the $R$ band used in the above-mentioned polarization instruments. The beam is simultaneously split by two dichroic mirrors into three electron multiplying CCD cameras (EMCCDs). The combination of the flux from the eight rotor positions using equations from Clarke \& Neumayer (2002) can be used to find the linear Stokes parameters, which were used to calculate the degree and angle of polarization. Finally, additional optical polarimetric data are reported here from the $70 \mathrm{~cm}$ AZT-8 telescope (Crimea) and the $40 \mathrm{~cm} \mathrm{LX-200} \mathrm{telescope}$ (St. Petersburg), both equipped with nearly identical imaging photometers-polarimeters (Larionov et al. 2008). Polarimetric observations were performed using two Savart plates rotated by $45^{\circ}$ relative to each another. By swapping the plates, the observer can obtain the relative Stokes $q$ and $u$ parameters from the two split images of each source in the field. Instrumental polarization was found via stars located near the object under the assumption that their radiation is unpolarized. The electric vector position angle (EVPA) was corrected for the $n \times 180^{\circ}$ ambiguity by minimizing the difference to the closest data point unless there is a gap of over 14 days.

\subsection{Infrared}

PKS 1510-089 is monitored by a number of IR instruments. We used the publicly available data in the $B, V, R, J$, and $K$ bands from the Small and Moderate Aperture Research Telescope System (SMARTS) instrument located at Cerro Tololo Interamerican Observatory (CTIO) in Chile. The data reduction and calibration is described in Bonning et al. (2012). We converted the magnitudes into flux units using Bessell et al. (1998) and corrected for the interstellar dust absorption following Schlafly \& Finkbeiner (2011).

The observations at Teide Observatory (Canary Islands) were obtained with the $1.52 \mathrm{~m}$ Carlos Sanchez Telescope (TCS), using the near-infrared camera CAIN during the nights of MJD 57162-57174. This camera is equipped with a $256 \times 256$ pixel NICMOS-3 detector providing a scale of $1^{\prime \prime} /$ pixel. Data were acquired in the three filters $J, H$, and $K$ s. Observations were performed using a 5-point dither pattern (repeated twice) in order to facilitate a proper sky background subtraction. At each point, the exposure time was about $1 \mathrm{~min}$, split into individual exposures of $10 \mathrm{~s}$ in the $J$ filter and $6 \mathrm{~s}$ in the $H$ and $K \mathrm{~s}$ filters to avoid saturation by sky brightness. Image reduction was performed with the caindr package under the IRAF environment ${ }^{6}$. Data reduction includes flat-fielding, sky subtraction, and the shift and combination of all frames taken in the same dither cycle. Photometric calibration was made based on field stars from the 2MASS catalog (Cutri et al. 2003). The photometric zero point was determined for each frame by averaging

\footnotetext{
6 Image Reduction and Analysis Facility, http : //iraf.noao. edu/
} 
the offset between the instrumental and the 2MASS magnitudes of the catalog. Deviant stars were excluded and typical errors remained below $5 \%$.

We also used IR photometry data obtained with the $1.2 \mathrm{~m}$ telescope of Mt Abu InfraRed Observatory (MIRO), India, mounted with the Near Infrared Camera and Spectrograph (NICS) equipped with $1024 \times 1024 \mathrm{HgCdTe}$ Hawaii array detector. The field of view is $8^{\prime} \times 8^{\prime}$ with a pixel scale of $0.5^{\prime \prime} /$ pixel. The observations on PKS 1510-089 were performed with a 4-position dither with offsets of 30 arcsec, keeping the comparison $\operatorname{stars}^{7} 1,2,3,4$, and 6 in the field of the source. The sky and dark contributions were removed using these dithered images and aperture photometry was performed using standard procedures under IRAF (see Banerjee \& Ashok 2012, for details on data reduction and analysis). The source magnitudes in the $J, H$, and $K \mathrm{~s}$ bands were calibrated using correction factors obtained using the weighted average of the standard values of comparison stars mentioned above.

\subsection{Radio}

Radio monitoring observations were performed with Metsähovi Radio Telescope operating at $37 \mathrm{GHz}$ frequency. The instrument and data reduction procedures are described in Teräesranta et al. (1998) and Aleksić et al. (2014).

The quasar PKS 1510-089 was observed within a sample of gamma-ray blazars that the Boston University (BU) blazar group monitors with the Very Long Baseline Array (VLBA) approximately monthly at $43 \mathrm{GHz}$ (the VLBA-BU-BLAZAR project). The observations of PKS 1510-089 are usually performed via nine short scans ( $\sim 5 \mathrm{~min}$ each) within a span of $7-8 \mathrm{~h}$. The data were calibrated at the VLBA DiFX correlator and reduced using the Astronomical Image Process System (AIPS) and Difmap software packages, as described in Jorstad et al. (2005). The calibrated data are available online ${ }^{8}$. We analyzed the data obtained from February 2015 to April 2016 (12 epochs). We modeled the total intensity images by components with circular Gaussian brightness distributions. For each component we determined flux density, distance and position angle (PA) with respect to the VLBI core ${ }^{9}$, and size. The $43 \mathrm{GHz}$ core is expected to be located at a distance of $\sim 6.5 \mathrm{pc}$ from the central engine of PKS 1510-089 (see Pushkarev et al. 2012; Aleksić et al. 2014). A map of the parsec scale jet of the quasar formed from 20 stacked images over six years of VLBA observations at $43 \mathrm{GHz}$ is plotted in Fig. 1 (individual images can be found at the BU blazar group website http://www.bu.edu/blazars/ VLBA_GLAST/1510.html). The image shows the VLBI core, which is the brightest compact feature located at the southeast end of the jet. The core is used as a reference point in the stacking procedure since it is assumed to be stationary. The stacked image reveals the full opening angle of the jet, as well as the location of the jet axis. As can be inferred from Fig. 1, the jet axis is along $\mathrm{PA} \sim-30 \mathrm{deg}$, while the projected opening angle is $\sim 60 \mathrm{deg}$. The core at $43 \mathrm{GHz}$ is only partially optically thick, which is supported by synchronous optical and radio core polarization variability in a number of blazars (D'Arcangelo et al. 2007; D'arcangelo et al. 2009). Figure 1 reveals a shift between the

\footnotetext{
7 https://www.lsw.uni-heidelberg.de/projects/ extragalactic/charts/1510-089.html

8 www.bu.edu/blazars/VLBAproject.htm

9 PA is measured starting from the positive direction of Declination axis $\left(\mathrm{PA}=0^{\circ}\right)$ increasing in the positive direction of Right Ascension axis $\left(\mathrm{PA}=90^{\circ}\right)$.
}

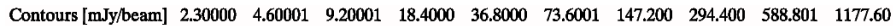

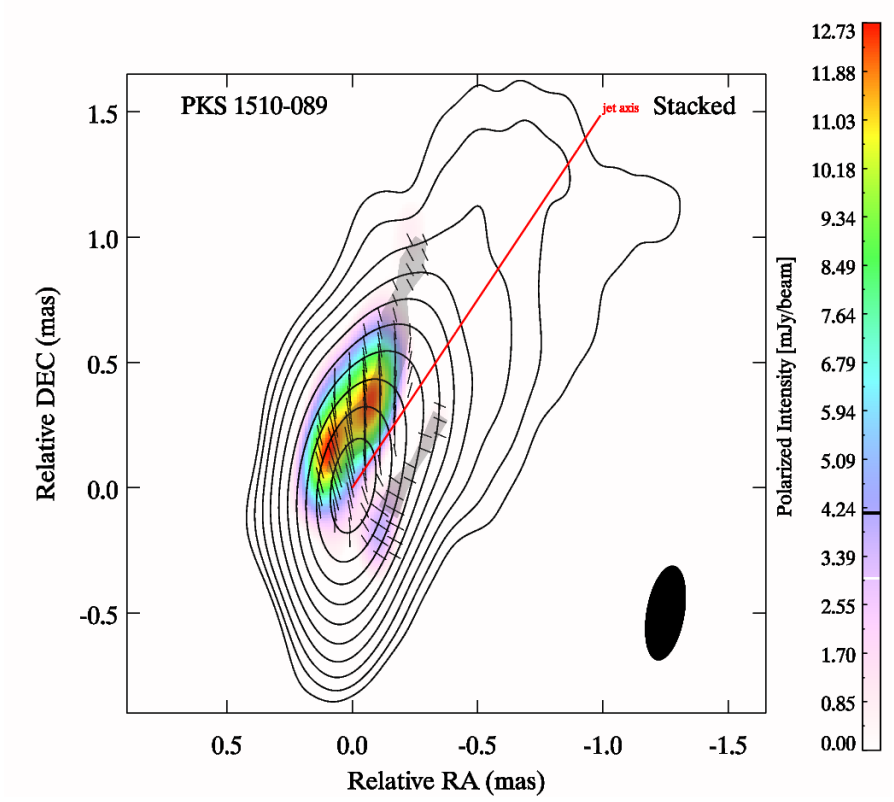

Fig. 1. Stacked map of $43 \mathrm{GHz}$ total (black contours) and polarized (color scale) intensity images of the inner pc-scale jet of PKS 1510-089 with the direction of electric field vector polarization denoted by black line segments (contour levels are indicated at the top, polarized flux levels are shown in the color bar to the right). All images have been convolved with the same Gaussian beam, shown in the lower right corner.

total and polarized intensity peaks and the complex structure of the polarized emission in the core. This favors the hypothesis that the core of PKS 1510-089 is a recollimation shock, which was inferred previously from the polarization structure in other blazars (e.g., Cawthorne et al. 2013).

\section{Results}

In Fig. 2 we show the multiwavelength light curve of PKS 1510-089 during the May 2015 outburst in the investigated period of MJD 57 151-57174. Following the observations of MAGIC, we define two observation periods: Period A (MJD 57 160-57 161) and Period B (MJD 57 164-57 166). The multiwavelength SED of both periods is investigated.

\subsection{MAGIC}

The MAGIC light curve (top panel of Fig. 2) shows clear variability, with the highest flux observed during the two nights of Period A. The hypothesis of constant flux during all five observation nights of MAGIC can be clearly rejected, with a chance probability of $7.7 \times 10^{-8}$. Even allowing for a $20 \%$ variable systematic uncertainty on individual night fluxes (motivated by the variable systematic uncertainty estimate given in Aleksić et al. 2016 b, rescaled to a softer source) we still obtain a small value of chance probability of $3.2 \times 10^{-4}$ that the flux is constant. The flux during Period $\mathrm{A}$ is $\sim 5$ times higher than that observed during the previous detection by MAGIC in 2012 (Aleksić et al. 2014). In the following observations during Period $B$, the VHE gamma-ray flux decreased to a level consistent with the detection in 2012 .

In order to search for a possible short time variability, we binned the light curve during Period A into 20 min bins (see Fig. 3). No variability is detected at such a time scale. Fitting the 


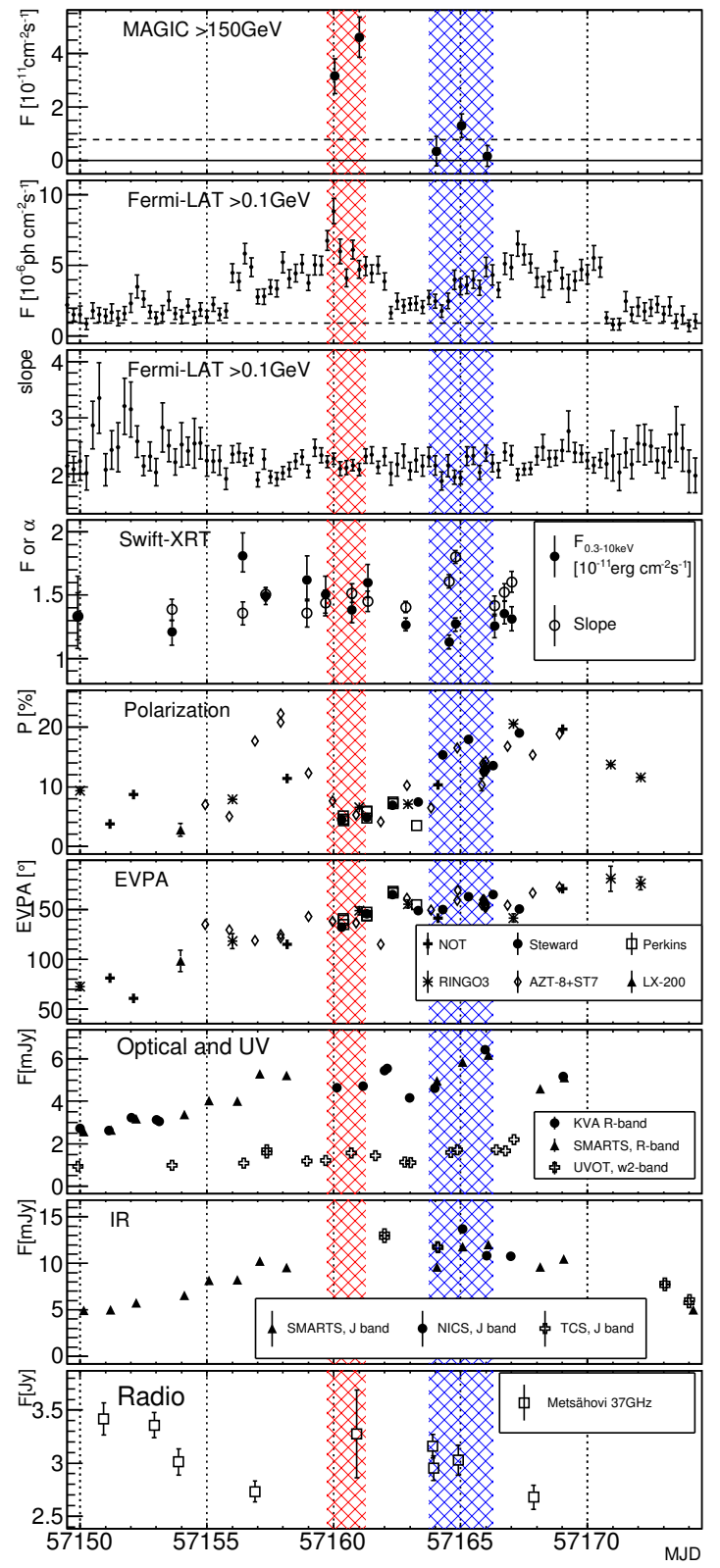

Fig. 2. Multiwavelength light curve of PKS 1510-089 during the May 2015 flare. From top to bottom: nightly gamma-ray flux above $150 \mathrm{GeV}$ from MAGIC (the dashed line shows the average emission in Feb.-Apr. 2012, Aleksić et al. 2014); Fermi-LAT flux above $0.1 \mathrm{GeV}$ in $6 \mathrm{~h}$ binning, and the corresponding spectral index (the dashed line shows the average emission from the 3FGL catalog, Acero et al. 2015); X-ray spectral flux (filled circles) and spectral index (empty circles) measured by Swift-XRT; polarization percentage and polarization angle measured by NOT, Steward, Perkins, RINGO3, AZT-8, and LX-200 (see legend); optical emission in $R$ band (KVA, SMARTS) and UV emission in $w 2-$ band (Swift-UVOT); IR emission in $J$ band (SMARTS, MIRO-NICS, TCS); radio observations by Metsähovi at $37 \mathrm{GHz}$. Data from IR to UV are corrected for Galactic absorption. The red and blue shaded regions show Period A and Period B, respectively, for which the spectral modeling is performed.

light curve with a constant flux hypothesis we obtain $\chi^{2} / N_{\text {d.o.f. }}=$ $5.9 / 7$. We estimate the maximum variability that can be hidden by the uncertainties of the measurement by computing for each 20 min light curve bin a $95 \%$ confidence level interval on the flux using the Rolke et al. (2005) prescription. We include a $20 \%$

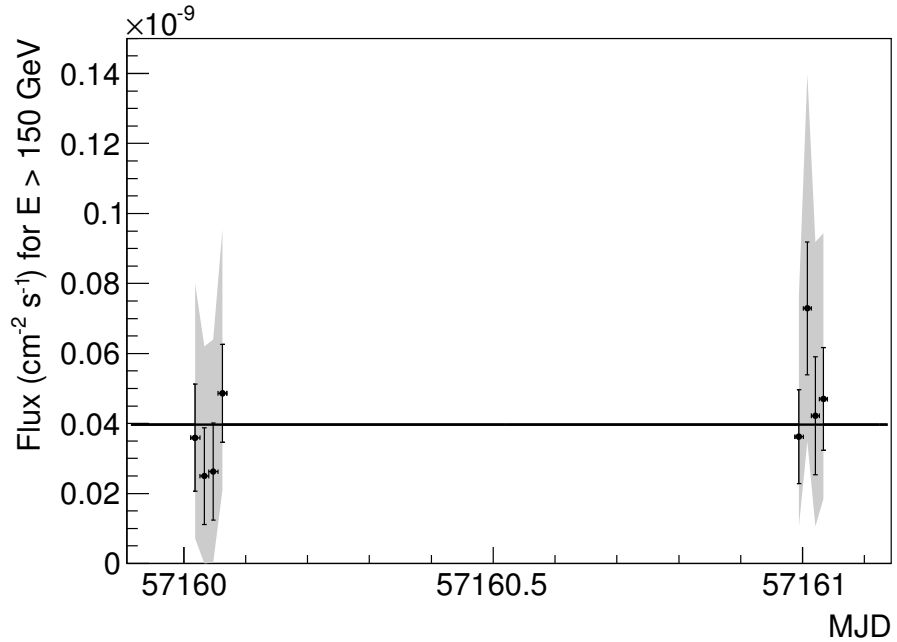

Fig. 3. Light curve above $150 \mathrm{GeV}$ obtained with the MAGIC telescopes during the flare in Period A. The fluxes are computed in $20 \mathrm{~min}$ bins. The black line shows the constant flux fit, $(4.0 \pm 0.5) \times 10^{-11} \mathrm{~cm}^{-2} \mathrm{~s}^{-1}$. The gray band shows the $95 \%$ confidence level interval allowing for $20 \%$ variable systematic uncertainty (see text for details).

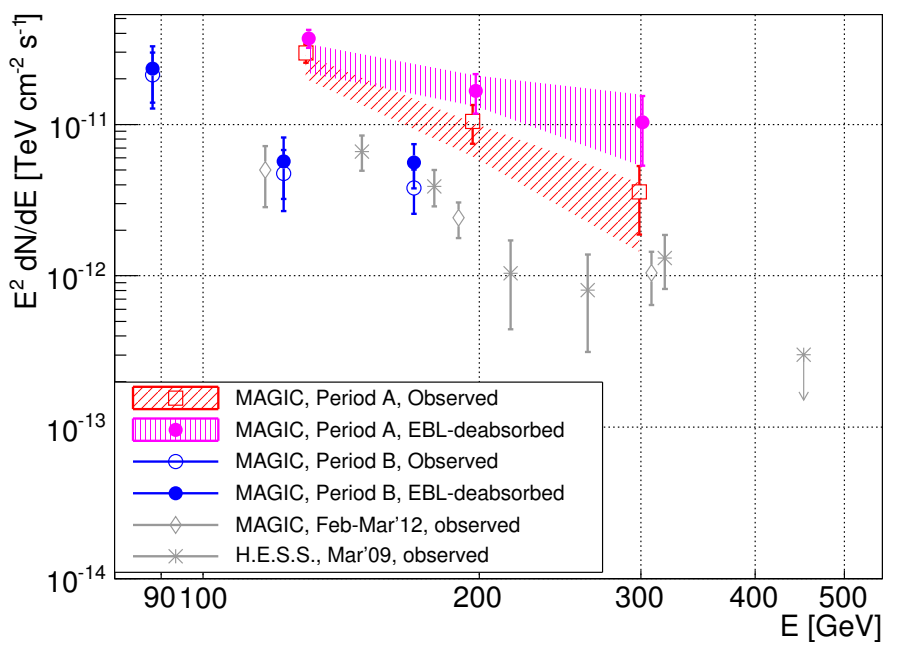

Fig. 4. Spectral energy distribution of PKS 1510-089 constructed from the MAGIC data gathered on MJD 57160 and 57161 (Period A). The observed spectrum is shown by red empty squares with inclined hashing, and the EBL-deabsorbed spectrum according to the Domínguez et al. (2011) model by magenta filled circles with vertical hashing. The hashing shows the uncertainty of the forward folding with a power law. The SED constructed from the data taken on MJD between 57164 and 57166 (Period B) is shown as empty (observed) and filled (EBL-deabsorbed) blue circles. For comparison, MAGIC measurements performed in Feb.-Mar. 2012 and H.E.S.S. measurements from Mar. 2009 (Abramowski et al. 2013) are shown as gray diamonds and stars, respectively.

variable systematic uncertainty in these calculations. By comparing the least constraining upper edge of the $95 \%$ confidence level interval with the average flux from those two nights we obtained that the flux did not increase by more than a factor of 3.5 on time scales of $20 \mathrm{~min}$.

For spectral analysis we combined the two nights of Period A. The obtained VHE gamma-ray spectrum of PKS 1510-089 is shown in Fig. 4. It can be described by a power law, $\mathrm{d} N / \mathrm{d} E=f \times(E / 200 \mathrm{GeV})^{-\alpha}\left[\mathrm{cm}^{-2} \mathrm{~s}^{-1} \mathrm{TeV}^{-1}\right]$, with $f=\left(1.96 \pm 0.55_{\text {stat }} \pm 0.36_{\text {syst }}\right) \times 10^{-10}$ and $\alpha=4.59 \pm 0.75_{\text {stat }}$. The spectral parameters are obtained using a forward folding 
method (Albert et al. 2007). The statistical uncertainty on the spectral index is much larger than the typical systematic uncertainty of \pm 0.15 of the observations performed with the MAGIC telescopes. The systematic uncertainty on the flux normalization does not include the uncertainty of the energy scale of MAGIC, which for this data set we estimate as $\lesssim 19 \%$, slightly larger than the $\$ 15 \%$ given in Aleksić et al. (2016b), due to the need of LIDAR correction of Calima-affected data. Correcting for the absorption of $\mathrm{TeV}$ gamma rays due to the interaction with the extragalactic background light according to the Domínguez et al. (2011) model, an intrinsic spectrum with normalization of $f_{\text {deabs }}=\left(4.2 \pm 1.0_{\text {stat }} \pm 0.76_{\text {syst }}\right) \times 10^{-10}$ and index $\alpha_{\text {deabs }}=3.17 \pm 0.80_{\text {stat }}$ are obtained. The spectral shape is marginally consistent (but with large uncertainties) with the previous measurements by H.E.S.S. (observed slope $5.4 \pm 0.7_{\text {stat }} \pm 0.3_{\text {syst }}$, Abramowski et al. 2013) and MAGIC (intrinsic slope $2.5 \pm 0.6_{\text {stat }}$, Aleksić et al. 2014).

For comparison, we have also reconstructed the average flux from the MAGIC measurements performed during Period B. The combination of weaker emission and observations performed during higher atmospheric transmission results in the energy range of the reconstructed spectrum shifted to lower energies. The observed flux in Period B is at a similar level as the one detected by MAGIC in 2012. We obtained the observed and intrinsic VHE spectral slopes of PKS 1510-089 during Period B of $4.75 \pm 0.62_{\text {stat }}$ and $4.33 \pm 0.75_{\text {stat }}$, respectively.

\subsection{Fermi-LAT}

The GeV gamma-ray flux of PKS 1510-089 is highly variable in the investigated period. A few individual flares are visible, with time scales of a few days. Owing to the shortterm gamma-ray variability, in order to get a spectrum comparable to the MAGIC observations, for SED analysis we selected the events observed by the LAT within $6 \mathrm{~h}$ centered on each of the MAGIC observations. We calculated two GeV spectra, which correspond to the two different states of the source contemporaneously to the MAGIC observations in Period A and Period B, respectively. The best description of the $\mathrm{GeV}$ spectrum measured by the LAT in Period A is a power law with spectral index $2.20 \pm 0.07$ and a flux above $100 \mathrm{MeV}$ of $(6.8 \pm 0.5) \times 10^{-6} \mathrm{ph} \mathrm{cm}^{-2} \mathrm{~s}^{-1}(\mathrm{TS}=842)$. During Period B, the Fermi-LAT measured spectrum is best described by a power law with a similar spectral index of $2.17 \pm 0.08$, but a significantly lower flux, $(3.7 \pm 0.3) \times 10^{-6} \mathrm{ph} \mathrm{cm}^{-2} \mathrm{~s}^{-1}$ $(\mathrm{TS}=564)$. For comparison, the $3 \mathrm{FGL}$ flux above $100 \mathrm{MeV}$ is $(0.94 \pm 0.01) \times 10^{-6} \mathrm{ph} \mathrm{cm}^{-2} \mathrm{~s}^{-1}$ (Acero et al. 2015). Therefore, PKS 1510-089 reached a factor of $\sim 7$ to 4 times its average flux over the first four years of Fermi-LAT observations during the two epochs for which the spectral analyses was performed. The GeV flux was, however, still smaller by a factor of about 2 than the daily peak flux observed in 2011 (Saito et al. 2015). The Fermi-LAT spectrum during both Period A and B was slightly harder than in the neighboring days (see also Sect. 3.5). In the case of Period B there is a weak hint of a spectral curvature. The likelihood ratio test gives $2.8 \sigma$ preference $(\Delta \mathrm{TS}=8.0$ for one additional degree of freedom) of the log-parabola shape over the power-law spectral model. The corresponding photon index can be described as $(2.05 \pm 0.10)+(0.21 \pm 0.09) \times \ln (E / 0.45 \mathrm{GeV})$.

To characterize the variability in the Fermi-LAT light curve we calculated the power density spectrum (PDS) for the 2015 flare epoch (MJD 57 143.9375-57 182.9375) with a $3 \mathrm{~h}$ binning and for a mission lifetime at the time of the analysis (MJD 54 682.655-57 484.655) with 1 day binning. In both cases

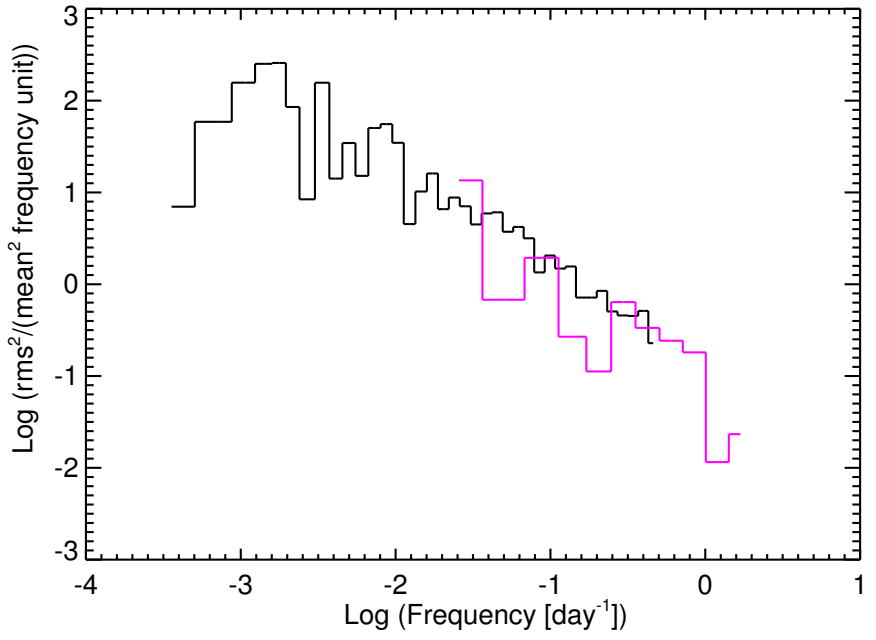

Fig. 5. Power density spectrum normalized to variance per frequency unit for the mission-long Fermi-LAT light curve (black) and for the 2015 flare epoch (magenta).

the flux calculation was performed in the $0.1-800 \mathrm{GeV}$ energy range.

The estimated white noise level, based on the data error values was subtracted from each PDS. The PDS is rebinned in logarithmic frequency intervals, and normalized to variance per frequency unit divided by the square of the mean flux. The PDS level, computed with this normalization, is similar for the 2015 flare and for the mission lifetime light curve (see Fig. 5). This suggests that the fractional variability is the same and presumably driven by the same variability process. The shape of the overall PDS can be described by a power law for frequencies above $0.01 \mathrm{day}^{-1}$. A power law fitted to the $0.007-0.5 \mathrm{day}^{-1}$ frequency range PDS gives an index of $1.14 \pm 0.07$ for full data set and $0.97 \pm 0.30$ for the 2015 flare. The uncertainty value of the fit is based on the scatter of the measurements with respect to the fitted line only and therefore may be underestimated. The powerlaw index is similar to that of other FSRQs (Ackermann et al. 2011). The stochastic nature of the variability together with the limited observation length lead to a large uncertainty in the PDS at the lowest frequencies.

\subsection{Swift-XRT}

The X-ray flux, measured by Swift-XRT, shows a gradual decrease in the observed flux during the studied period. Except for the first point at MJD 57 153.6, which happened before the two large Fermi-LAT flares, the X-ray flux can be much better described by an exponential decline $\left(\chi^{2} / N_{\text {d.o.f. }}=22.6 / 10\right)$ than a constant value $\left(\chi^{2} / N_{\text {dof }}=47.3 / 11\right)$. Similarly, the corresponding X-ray spectral indices are better described by a linear softening of the spectrum $\left(\chi^{2} / N_{\text {dof }}=41.0 / 10\right)$ than a constant $\left(\chi^{2} / N_{\text {d.o.f. }}=49.9 / 11\right)$.

The flux is marginally higher during MJD 57 156-57 162, when a broad Fermi-LAT flare occurred. XRT observations on MJD 57 166-57 168, during the following broad Fermi flare did not show a clear increase in the X-ray flux. For spectral analysis we combined the four pointings taken during $\mathrm{Pe}$ riod $\mathrm{A}$, each of them within $6-8 \mathrm{~h}$ of the MAGIC observations. The X-ray spectrum in Period A can be marginally well described $\left(\chi^{2} / N_{\text {d.o.f. }}=71 / 53\right)$ with a power law with an index of $1.48 \pm 0.05$. Swift-XRT observations performed during Period $\mathrm{B}$ resulted in a spectrum that can be well fitted with 
a power law with a significantly softer index of $1.70 \pm 0.04$ with $\chi^{2} / N_{\text {d.o.f. }}=110 / 89$, or by a curved spectrum with an index of $2.23 \pm 0.14-(0.366 \pm 0.093) \times \log (E / 0.3 \mathrm{keV})$ with $\chi^{2} / N_{\text {dof }}=96 / 88$.

\subsection{IR, optical, and UVOT}

The optical-UV SED of PKS 1510-089 consists of an unpolarized, quasi-stable accretion disk component and a non-thermal jet emission. The variability and polarization of the jet component might be diluted by the accretion disk component. The effect is strongest in the $U V$ bands, and weak in the $R$ band where the polarization is measured. It does not affect the timing of the observed polarization variability.

The optical emission of PKS 1510-089 during the investigated period is clearly variable (with a factor of 2 difference between the lowest and highest flux). The optical variability does not strictly follow the gamma-ray variability. In the optical $R$-band, and to a lesser extent also in the UV $w 2$-band, the flux slowly increased throughout May 2015. Similarly to the optical range, the IR flux doubled during the $\sim 8$ days before Period A. At the end of the observation period it returned to the pre-flare state.

Throughout the investigated period, a smooth rotation of optical EVPA by $\sim 100^{\circ}$ occurred. The rotations of optical polarization angle also accompanied the 2009 and 2012 gamma-ray flaring states (Marscher et al. 2010; Aleksić et al. 2014). However, the rotations of the EVPA seem very common in PKS 1510-089, e.g., recent work by Jermak et al. (2016) also identified a rotation in the 2011 data; therefore, further data are needed to firmly associate them with the emission of VHE gamma rays. The low percentage of polarization seems to be typical for this source (Jermak et al. 2016). Indeed, during Period A the polarization percentage is low $(\sim 5 \%)$. It triples between period $A$ and $B$. In the few days before Period A (i.e., during the raising part of the Fermi-LAT flare that culminated with detection of VHE gammaray emission by MAGIC), a higher polarization was also observed. The polarization behavior during the 2015 flaring period agrees with what is expected from a knot following a spiral path through a mainly toroidal magnetic field (Marscher et al. 2010). An alternative explanation might be the light travel time effects within an axisymmetric emission region pervaded by a predominately helical magnetic field (Zhang et al. 2015). The evolution of the polarization is probably further complicated by the superposition of individual flares seen in Fermi-LAT.

\subsection{Radio}

PKS 1510-089 shows moderate variability in the Metsähovi observations performed at $37 \mathrm{GHz}$ in May 2015. The sampling is, however, rather sparse, and especially the local peaks of the GeV flux are mostly not covered by the observations. The observations on MJD 57161 during Period A are burdened with a larger uncertainty, due to adverse atmospheric conditions.

Figure 6 shows the light curve of the core from February 2015 to April 2016. The light curve reveals a significant gradual increase in flux in the second half of 2015 that is most likely connected with a disturbance (knot $K 15)$ detected in the VLBA images starting in December 2015 (Fig. 7). Knot $K 15$ is bright and relatively slow, with an apparent speed $\beta_{\text {app }}=(5.3 \pm 1.4) c$. According to currently available data, the knot was ejected on MJD $57230 \pm 52$ (see Fig. 8). A similar behavior has also been observed during a high gamma-ray state in Feb.-Apr. 2012,

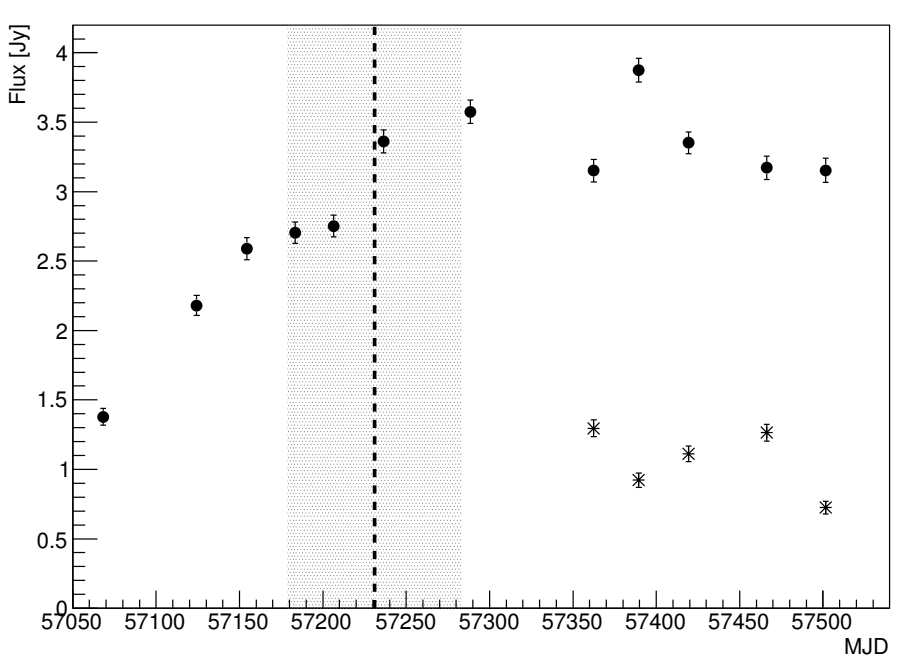

Fig. 6. VLBA light curves of the core (circles) and knot $K 15$ (stars) at $43 \mathrm{GHz}$. The vertical dashed line and gray shaded region show the zero separation epoch of $K 15$ and its uncertainty.

when the emergence of a new radio knot $(K 12)$ from the core was associated with a VHE outburst (Aleksić et al. 2014). The large uncertainty in the ejection time of $K 15$ does not allow us to associate it firmly with a particular $\mathrm{GeV}$ flare as the source showed activity in Fermi-LAT close to the time of $K 15$ separation (e.g., the hard flare on MJD 57245 in Fig. 9). Interestingly, the position angle of $K 15$ is $\sim+50^{\circ}$, while the typical projected direction of the pc-scale jet is $\sim-30^{\circ}$ (see Fig. 1), where seven knots have been observed to date (see, e.g., Jorstad et al. 2005; Aleksić et al. 2014). On the other hand, knot $K 11$, ejected in October 2011 (before the 2012 VHE outburst), had a similar PA to the most recently observed one for $K 15$ close to the core, but turned toward the usual pc-scale jet direction a few months later. We note that $K 15$ is a factor of a few times slower than $K 11$, and so may eventually follow a similar trajectory. The slower apparent speed and brighter flux of $K 15$ suggest that the velocity vector of this disturbance is closer to the line of sight than $K 11$, causing it to have a higher Doppler factor.

\section{Modeling the spectral energy distribution}

The gamma-ray emission of FSRQs is usually explained as the effect of the inverse Compton scattering of electrons on a radiation field external to the jet (see, e.g., Sikora et al. 1994; Ghisellini et al. 2010), the so-called external Compton (EC) scenario. The radiation field can originate from the accretion disk, broad-line region (BLR) or the dust torus (DT). This scenario has been applied to explain the emission of PKS 1510-089 in its previous flaring episodes (Abdo et al. 2010). The origin of the radiation field is closely connected to the location of the emission region. Moreover, the observed VHE gamma rays escaping from the emission region suggest that the emission region is located outside the BLR in order to escape the absorption by $\mathrm{e}^{+} \mathrm{e}^{-}$ pair production process on BLR photons (Abramowski et al. 2013; Aleksić et al. 2014). Dotson et al. (2015) investigated the 2009 GeV flares of PKS 1510-089 using the energy dependence of the flare decay time as the diagnostic for the emission zone location. They claimed that two of the flares happened around the distance of the DT, while the other two came from the vicinity of VLBI core. On the other hand, the modeling of $\mathrm{GeV}$ flares seen from PKS 1510-089 in 2011 placed the emission region at the distance of $0.3-3$ pc from the black hole, with 


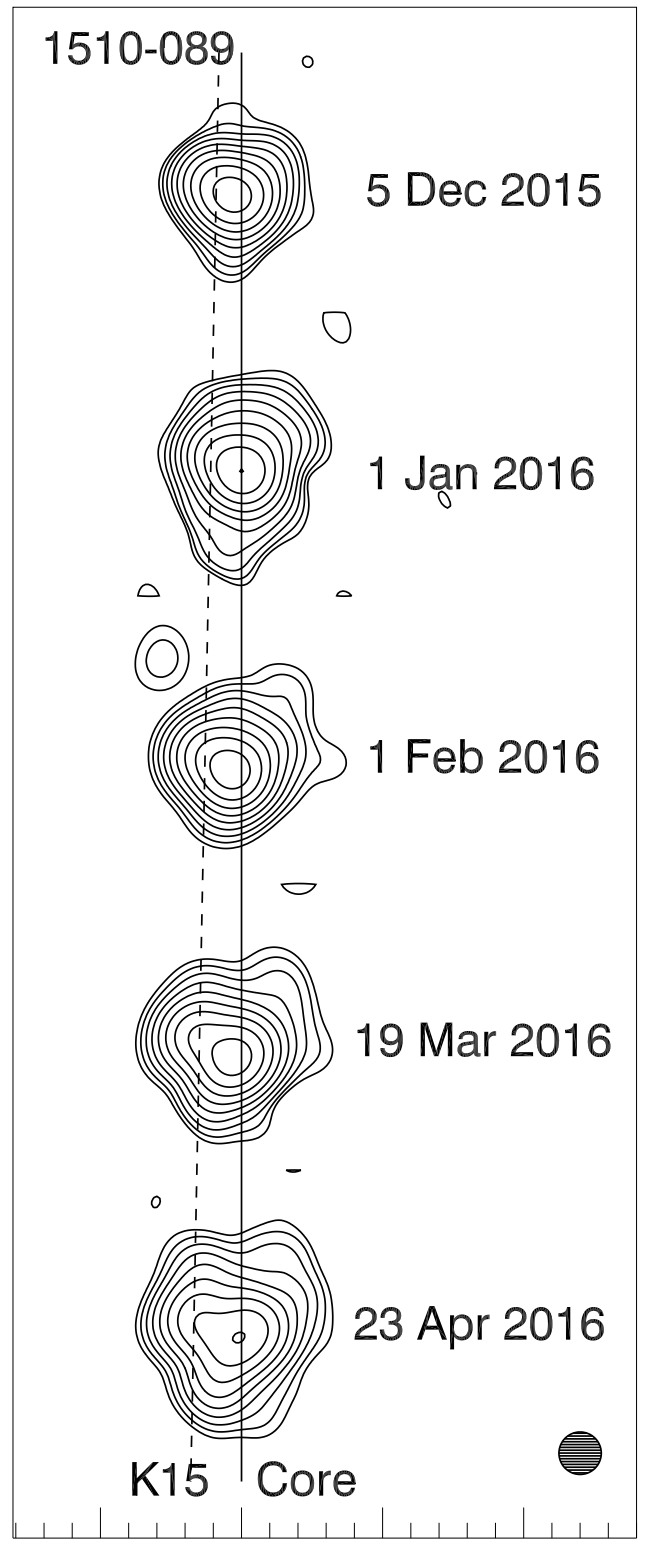

\section{$\begin{array}{llll}0.5 & 0.0 & -0.5 & -1.0\end{array}$}

\section{Rel RA Milliarcseconds}

Fig. 7. Total intensity images of the PKS1510-089 core region at $43 \mathrm{GHz}$, with a global peak intensity of $I_{\text {peak }}=3.566 \mathrm{Jy} /$ beam and 0.15 mas FWHM circular Gaussian restoring beam (bottom right circle). The solid and dashed lines designate positions of the VLBI core and $K 15$, respectively, across the epochs.

the EC process happening on a mixture of BLR and DT radiation fields (Saito et al. 2015). A similar location ( 1 pc from the black hole) with EC mainly on DT was invoked to explain the high optical and gamma-ray state observed at the beginning of 2012 (Aleksić et al. 2014). The broadband emission could be also explained by a much more distant region $(\sim 20 \mathrm{pc})$ for a twozone model where the jet consists of the inner spine and outer sheath layer (Aleksić et al. 2014; MacDonald et al. 2015).

In Fig. 10 we present the two spectral energy distributions of PKS 1510-089 constructed from the data taken in Periods A and $\mathrm{B}$, corresponding to high and low gamma-ray flux, respectively. As can be seen, most of the flux variation $(\approx$ a factor of

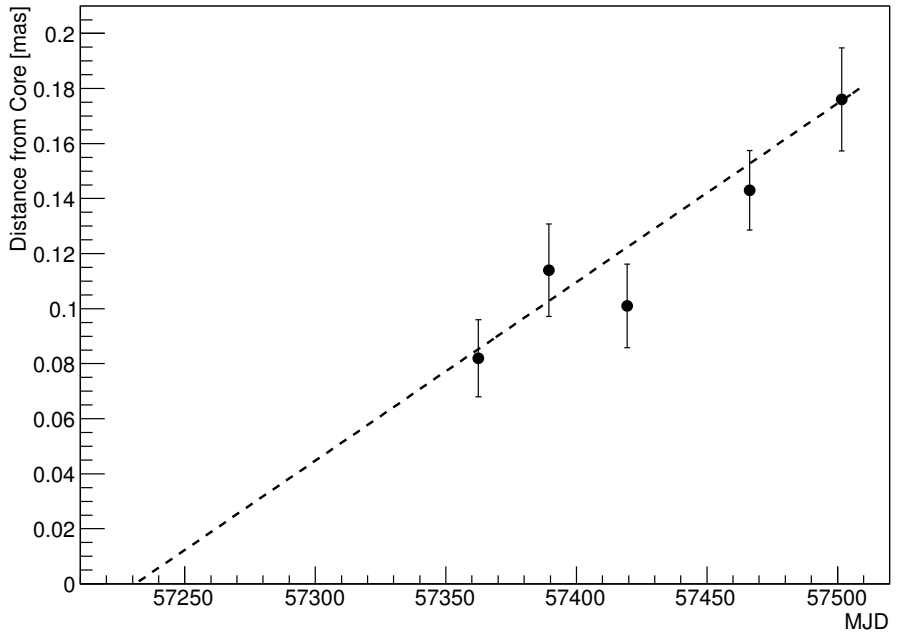

Fig. 8. Separation of knot $K 15$ from the core.
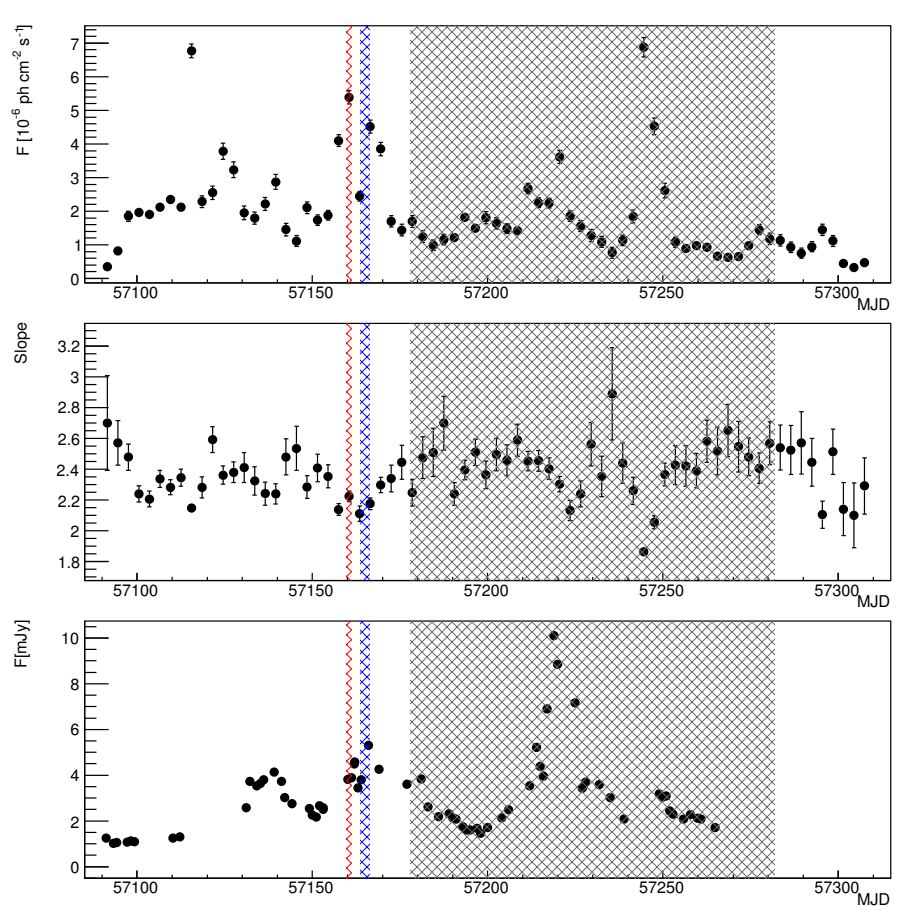

Fig. 9. Fermi-LAT light curve above $0.1 \mathrm{GeV}$ (top panel) binned in 3 -day intervals and the corresponding evolution of spectral index (middle panel) and the optical $R$-band flux observed by KVA (bottom panel). The red and blue hashed bands show Period A and B, respectively, while the gray band shows the $1 \sigma$ uncertainty on the $K 15$ knot separation time.

2-3) occurs in the Fermi-LAT and MAGIC bands. The lowenergy flux (optical, $\mathrm{X}$-rays) is almost constant between the two periods. Remarkably, the high-energy peak during the period B has a very similar level to the 2012 high state, even though the IR-UV emission is a factor of $\sim 3$ higher.

We model these SEDs of PKS 1510-089 in the framework of the same one-zone model, as discussed in Aleksić et al. (2014). To explain the sub-TeV emission observed by MAGIC we assume that the emission region is located beyond the BLR radius where the external photon field is dominated by the thermal IR radiation of the DT.

For the setup of the model we assume the scaling laws and the prescriptions given in Ghisellini \& Tavecchio (2009). 


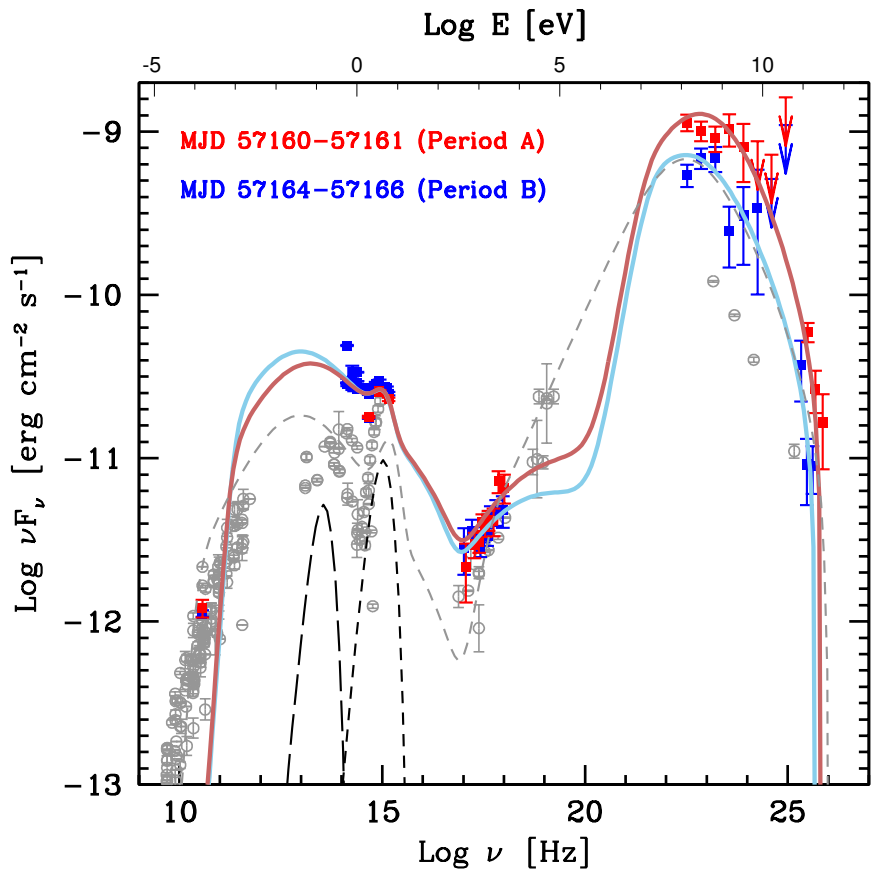

Fig. 10. Multiwavelength spectral energy distribution of PKS 1510-089 in Periods A (red symbols) and B (blue symbols). The red and the blue curves show the result of the emission model for the two periods. The black dashed and long-dashed lines show the adopted emission for the accretion disk and the dusty torus, respectively. For comparison, the dashed gray line shows the model derived for the SED in 2012 (from Aleksić et al. 2014). Historical measurements (ASDC, see http://www.asdc.asi.it/) are shown as gray points.

Specifically, the radius of the BLR is given by $R_{\mathrm{BLR}}=$ $10^{17} L_{\mathrm{d}, 45}^{1 / 2} \mathrm{~cm}$ and that of the torus by $R_{\mathrm{IR}}=2.5 \times 10^{18} L_{\mathrm{d}, 45}^{1 / 2} \mathrm{~cm}$, where $L_{\mathrm{d}, 45}^{1 / 2}$ is the accretion disk luminosity in units of $10^{45} \mathrm{erg} \mathrm{s}^{-1}$. We calculate the IR radiation energy density assuming that a fraction $f_{\mathrm{IR}}=0.6$ of the disk radiation is intercepted and reprocessed by the torus heated to $1000 \mathrm{~K}$. Similarly, the BLR intercepts $f_{\mathrm{BLR}}=0.1$ of the disk radiation. With these prescriptions, we note that the energy densities of the BLR and torus radiation fields do not depend on the disk luminosity since they depend on the constant ratio $L_{\mathrm{disk}} / R_{\mathrm{BLR} \text { or IR }}^{2}$. Assuming the same disk luminosity $L_{\mathrm{disk}}=6.7 \times 10^{45} \mathrm{erg} \mathrm{s}^{-1}$ as in Aleksić et al. (2014) the scaling law of Ghisellini \& Tavecchio (2009) (based on reverberation mapping measurements of BLR size; see, e.g., Bentz et al. 2009) allows us to infer a BLR radius of $R_{\mathrm{BLR}}=2.6 \times 10^{17} \mathrm{~cm}$.

We fix the distance of the emission region from the base of the jet to $r=6 \times 10^{17} \mathrm{~cm}$. The emission region is filling the whole cross section of the jet, which for an assumed jet semiaperture angle $\theta_{\mathrm{j}}=0.047 \mathrm{rad}$, results in the emission region radius $R=2.8 \times 10^{16} \mathrm{~cm}$. This size for the emission region is in line, even for moderate values of the Doppler factor, with the constraints set by the time scale variability of a few days observed by MAGIC.

The apparent superluminal motion of radio component K15 puts limits on $\Gamma \gtrsim 5$. The large uncertainty in the separation time from the radio core does not allow us to firmly associate such limits with the speed of the emission region responsible for the emission in investigated Periods A and B. Moreover, the apparent speed is measured over a much longer time scale, when the emission region might have decelerated. On the other hand, the beaming of the emission is constrained by the observed luminosity of the dominating high-energy peak. It can be estimated as $L_{\mathrm{EC}}=\frac{4}{3} \sigma_{\mathrm{T}} c U_{\mathrm{ext}}^{\prime} n_{\mathrm{e}}\left\langle\gamma^{2}\right\rangle V \delta^{4}$, where $U_{\mathrm{ext}}^{\prime}$ is the energy density of the external radiation field measured in the frame of reference of the blob, $n_{\mathrm{e}}$ is the number density of the electrons, $V=4 / 3 \pi R^{3}$ is the volume of the emission region measured in its own frame of reference, and $\delta$ is the Doppler factor of the blob. The average squared Lorentz factor of the electrons $\left\langle\gamma^{2}\right\rangle$ can be approximated as $\gamma_{\mathrm{b}}$ if the distribution starts from $\gamma_{\text {min }} \approx 1$ and follows an index of 2 up to the break of $\gamma_{\mathrm{b}}$. Assuming $\Gamma \approx \delta$, the total kinetic power of a jet composed of cold protons with number density of $n_{\mathrm{p}}$ is $P_{\text {jet }}=\pi R^{2} n_{\mathrm{p}} m_{\mathrm{p}} c^{3} \Gamma^{2}$. Combining the two formulas, for a jet with $n_{\mathrm{e}} \approx n_{\mathrm{p}}$ we obtain $P_{\text {jet }}=$ $1.3 L_{\mathrm{EC}}\left(U_{\mathrm{ext}}^{\prime} / 0.1 \mathrm{erg} \mathrm{cm}^{-3}\right)^{-1}\left(\gamma_{\mathrm{b}} / 10^{3}\right)^{-1}\left(R / 10^{17} \mathrm{~cm}\right)^{-1}(\Gamma / 10)^{-2}$.

The observed luminosity of $\sim 4 \times 10^{47} \mathrm{erg} \mathrm{s}^{-1}$ of the EC peak requires the jet Lorentz factor to be at least 10 even for the case of $P_{\text {jet }}=10 L_{\text {disk }}$. For the modeling we apply the same values as used in Aleksić et al. (2014) for the jet bulk Lorentz factor $\Gamma=20$ and $\delta=25$. For such a bulk Lorentz factor the assumed jet semi-aperture angle of $0.047 \mathrm{rad}$ is broader than $0.01(\Gamma / 20)^{-1}$ suggested by radio observations (see, e.g., Jorstad et al. 2005). The radio observations are sensitive to the jet opening angle at a distance equal to or greater than the location of the radio core, i.e., a few pc from the base of the jet. On the other hand, the emission region assumed in the modeling is located closer to the base of the jet, where the jet opening angle can be larger, as observed, for example in the case of the M87 radio galaxy (Asada \& Nakamura 2012).

Having fixed these values, the free parameters of the model are only the intensity of the magnetic field $B$ and those describing the electron energy distribution. Hence, the observed variability according to this scenario is caused by changes in the conditions of the plasma flowing through the shock region. Since we assume that the emission occurs outside the intense radiation field of the BLR, the IC emission and the radiative losses of the emitting electrons are dominated by the scattering of the IR radiation field of the torus. As the energy density of this radiation field is relatively low, the cooling of the electrons is not very effective. A simple calculation shows that the Lorentz factor at which the cooling time equals the dynamical time $R / c$ is on the order of $\gamma_{\text {cool }} \approx 940 R_{16}^{-1}(\Gamma / 20)^{-2}$. If we assume that the electrons are injected starting from $\gamma_{\text {inj,min }}=1$, with a power-law distribution with slope $n_{\text {inn }}$, in equilibrium we would expect a break at $\gamma_{\text {cool }}$, above which the slope of the distribution would steepen to $n_{\text {inj }}+1$. However, such a break could not properly describe the $\mathrm{SED}$, since the required break (estimated using the X-ray and the MAGIC slopes) is larger (even taking into account KleinNishina effects on the spectrum in the MAGIC energy range). To reproduce the SED we therefore assume a scenario in which the electron energy distribution is a double broken power law; there is a cooling break at $\gamma_{\mathrm{c}}$, and a second break (most probably connected with the acceleration process) at $\gamma_{\mathrm{b}}$. The particles are injected into the emission region with a broken power-law energy distribution with slopes $n_{1}^{\prime}=1$ and $n_{2}^{\prime}=2.7$, and break Lorentz factor $\gamma_{\mathrm{b}}$. In equilibrium conditions, the electron energy distribution displays three power laws with slopes $n_{0}=n_{1}^{\prime}$ from $\gamma_{\text {min }}$ to the cooling electron Lorentz factor $\gamma_{\mathrm{c}}, n_{1}=n_{1}^{\prime}+1=2$ from $\gamma_{\mathrm{c}}$ to $\gamma_{\mathrm{b}}$, and $n_{2}=n_{2}^{\prime}+1=3.7$ above $\gamma_{\mathrm{b}}$ (see, e.g., Ghisellini et al. 2002). We note that similar hard spectra have been also postulated when modeling blue blazars (Ghisellini et al. 2012).

The values of these parameters required to reproduce the SEDs are listed in Table 1. In fact, the difference in the emission between Period A and B can be explained with a relatively small 
A\&A 603, A29 (2017)

Table 1. Input model parameters for the models of Periods A (MJD 57 160-57 161) and B (57 164-57 166) of PKS $1510-089$ in Fig. 10.

\begin{tabular}{cccccccccc}
\hline \hline & $\gamma_{\min }$ & $\gamma_{\mathrm{c}}$ & $\gamma_{\mathrm{b}}$ & $\gamma_{\max }$ & $n_{0}$ & $n_{1}$ & $n_{2}$ & $B$ & $K$ \\
& {$[1]$} & {$[2]$} & {$[3]$} & {$[4]$} & {$[5]$} & {$[6]$} & {$[7]$} & {$[8]$} & {$[9]$} \\
\hline Period A & 1 & 150 & 800 & $4 \times 10^{4}$ & 1 & 2 & 3.7 & 0.23 & $3.0 \times 10^{4}$ \\
Period B & 1 & 150 & 500 & $3 \times 10^{4}$ & 1 & 2 & 3.7 & 0.34 & $2.6 \times 10^{4}$ \\
\hline
\end{tabular}

Notes. Columns [1], [2], [3], and [4]: minimum, cooling break, acceleration break, and maximum electron Lorentz factor, respectively. [5], [6], and [7]: slope of the electron energy distribution below $\gamma_{c}$, between $\gamma_{c}$ and $\gamma_{b}$, and above $\gamma_{b}$. [8]: magnetic field [G]; [9]: normalization of the electron distribution in units of $\mathrm{cm}^{-3}$.

change in the fit parameters, namely a slightly stronger magnetic field and lower maximum and break energies of the electrons

The model discussed here has some caveats. As typically happens in blazar modeling, the radio points overshoot the model line, which has a strong low-energy cutoff due to synchrotron self-absorption. This emission is normally thought to occur from much larger regions farther along the jet. Moreover, the variations in the optical emission occur on longer time scales than the flares observed in GeV. Hence, additional optical emission might be produced by the high-energy electrons swept with the flow farther along the jet (up to a few pc from the base of the jet). In those regions the external radiation field density would be too low to efficiently produce high-energy photons via the inverse Compton process, turning synchrotron emission into the dominant radiation process. Even though electrons can suffer adiabatic energy losses, the total observed synchrotron radiation might still slowly increase, due to aggregation of electrons from multiple individual flares. In fact, if the electrons reach the radio core (and beyond) located at the distance of $d_{\text {core }}$ they could be responsible for the emission of a new radio knot. The separation time of such a knot from the core could be estimated to occur $(1+z) d_{\text {core }} /(c \delta \Gamma)=21\left(d_{\text {core }} / 6.5 \mathrm{pc}\right)(\delta / 25)^{-1}(\Gamma / 20)^{-1}$ days after the gamma-ray flare. The extent of the core might shorten the time delay before the knot-core interaction starts. We encourage further trials of modeling of the observed high state with scenarios employing emission from a greater length of the jet than a single active region.

\section{Discussion and conclusions}

Using the MAGIC telescopes data, we detected enhanced VHE gamma-ray emission from the direction of PKS 1510-089 during the high optical and GeV state of the source in May 2015. It was the first time that VHE gamma-ray variability was detected for this source. The spectral shape is, however, consistent within the statistical uncertainties with the previous measurements of the source. During May 2015 the IR through UV data showed a gradual increase in flux, while the flux in the X-ray range was slowly decreasing.

The May 2015 data revealed, similarly to the 2012 data, that the enhanced VHE gamma-ray emission occurred during the rotation of the optical polarization angle. Also, similarly to other gamma-ray flares, an ejection of a new radio component was observed; however, it had an unusual position angle. This suggests that the association of VHE gamma-ray emission with the rotation of EVPA and ejection of a new radio component might be a common feature of PKS 1510-089.

The source was modeled with the external Compton scenario. The evolution of the state of the source from the VHE gamma-ray flare to a lower emission (at the 2012 high state level reported in Aleksić et al. 2014) can be explained by relatively small changes in the conditions of the plasma flowing through the emission region. The presented scenario is, however, only one possible solution. As discussed in Aleksić et al. (2014), if we assume that the VHE flaring is indeed connected to the ejection of the new component (in this case $K 15$ ) from the VLBA core and the rotation of the optical polarization angle, it would be natural to assume a single emission region located far outside the dusty torus. In this case the seed photons could be provided by the sheath of the jet and this scenario has been shown to provide a feasible description of the previous flaring epochs of PKS 1510-089 (Aleksić et al. 2014; MacDonald et al. 2015). The VHE gamma-ray variability with time scale $\tau$ seen during the 2015 outburst puts constraints on the size, and therefore also on the location of the emission region. Assuming that the spine of the jet fills a significant fraction of the jet (as in Aleksic et al. 2014), the location of the emission region cannot be farther than $d=\tau \delta c /\left((1+z) \theta_{\mathrm{j}}\right)=2.7(t / 3$ days $)(\delta / 25)\left(\theta_{\mathrm{j}} / 1^{\circ}\right)^{-1} \mathrm{pc}$. Therefore, a high Doppler factor and a narrow jet are needed in order to place the emission region at the radio core. In fact, such low values of the jet extension, $(0.2 \pm 0.2)^{\circ}$ (Jorstad et al. 2005) and $0.9^{\circ}$ (Pushkarev et al. 2009) at the radio core are reported by the radio observations. Alternatively, the inner spine can be much narrower than the whole jet, as suggested by MacDonald et al. (2015).

To further study the connection of VHE emission with events at lower frequencies, long-term monitoring data are needed and this question will be addressed in a future publication. With the detection of this flare from PKS 1510-089, VHE gammaray variability (on time scales varying from tens of minutes to days) has been observed in all FSRQs known in VHE gamma rays. Fast-varying VHE gamma-ray emission is common among the brightest gamma-ray FSRQs. As it seems that most of the gamma-ray FSRQs can only be detected during these flares, it is not surprising at all that only a handful have been detected in VHE gamma-rays.

Acknowledgements. The MAGIC collaboration would like to thank the Instituto de Astrofísica de Canarias for the excellent working conditions at the Observatorio del Roque de los Muchachos in La Palma. The financial support of the German BMBF and MPG, the Italian INFN and INAF, the Swiss National Fund SNF, the ERDF under the Spanish MINECO (FPA2015-69818P, FPA2012-36668, FPA2015-68278-P, FPA2015-69210-C6-2-R, FPA201569210-C6-4-R, FPA2015-69210-C6-6-R, AYA2013-47447-C3-1-P, AYA201571042-P, ESP2015-71662-C2-2-P, CSD2009-00064), and the Japanese JSPS and MEXT is gratefully acknowledged. This work was also supported by the Spanish Centro de Excelencia "Severo Ochoa" SEV-2012-0234 and SEV-2015-0548, and Unidad de Excelencia "María de Maeztu” MDM-2014-0369, by grant 268740 of the Academy of Finland, by the Croatian Science Foundation (HrZZ) Project 09/176 and the University of Rijeka Project 13.12.1.3.02, by the DFG Collaborative Research Centers SFB823/C4 and SFB876/C3, and by the Polish MNiSzW grant 745/N-HESS-MAGIC/2010/0. The Fermi LAT Collaboration acknowledges generous ongoing support from a number of agencies and institutes that have supported both the development and the operation of the LAT, as well as scientific data analysis. These include the National Aeronautics and Space Administration and the Department of Energy in the United States; the Commissariat à l'Énergie Atomique and the Centre National de la Recherche Scientifique/Institut National de Physique Nucléaire et de Physique des Particules in France; the 
Agenzia Spaziale Italiana and the Istituto Nazionale di Fisica Nucleare in Italy; the Ministry of Education, Culture, Sports, Science and Technology (MEXT), High Energy Accelerator Research Organization (KEK), and Japan Aerospace Exploration Agency (JAXA) in Japan; and the K. A. Wallenberg Foundation, the Swedish Research Council, and the Swedish National Space Board in Sweden. The Metsähovi team acknowledges the support from the Academy of Finland to our observing projects (numbers 212656, 210338, 121148, and others). This paper has made use of up-to-date SMARTS optical/near-infrared light curves that are available at www . astro.yale.edu/smarts/glast/home.php. MIRO is operated by Physical Research Laboratory, Ahmedabad with support from Dept. of Space, government of India. This article is based on observations made with the $1.5 \mathrm{~m}$ telescope Carlos Sánchez operated by the Instituto de Astrofisica de Canarias in the Teide Observatory. The data presented here were obtained in part with ALFOSC, which is provided by the Instituto de Astrofisica de Andalucia (IAA) under a joint agreement with the University of Copenhagen and NOTSA. The Liverpool Telescope is operated on the island of La Palma by Liverpool John Moores University in the Spanish Observatorio del Roque de los Muchachos of the Instituto de Astrofisica de Canarias with financial support from the UK Science and Technology Facilities Council. St. Petersburg University team acknowledges support from Russian RFBR grant 15-02-00949 and St.Petersburg University research grant 6.38.335.2015. The BU group acknowledges support by NASA under Fermi Guest Investigator grants NNX11AQ03G and NNX14AQ58G. The VLBA is an instrument of the National Radio Astronomy Observatory. The National Radio Astronomy Observatory is a facility of the National Science Foundation operated under cooperative agreement by Associated Universities, Inc. We would like to thank the anonymous referee for the comments on the manuscript.

\section{References}

Abdo, A. A., Ackermann, M., Agudo, I., et al. 2010, ApJ, 721, 1425 Acero, F., Ackermann, M., Ajello, M., et al. 2015, ApJS, 218, 23 Acero, F., Ackermann, M., Ajello, M., et al. 2016, ApJS, 223, 26 Ackermann, M., Ajello, M., Allafort, A., et al. 2011, ApJ, 743, 171 Ahnen, M. L., Ansoldi, S., Antonelli, L. A., et al. 2015, ApJ, 815, L23 Albert, J., Aliu, E., Anderhub, H., et al. 2007, NIM A, 583, 494 Aleksić, J., Antonelli, L. A., Antoranz, P., et al. 2011, ApJ, 730, L8 Aleksić, J., Ansoldi, S., Antonelli, L. A., et al. 2014, A\&A, 569, A46 Aleksić, J., Ansoldi, S., Antonelli, L. A., et al. 2016a, Astropart. Phys., 72, 61 Aleksić, J., Ansoldi, S., Antonelli, L. A., et al. 2016b, Astropart. Phys., 72, 76 Arnold, D. M., Steele, I. A., Bates, S. D., Mottram, C. J., \& Smith, R. J. 2012, Proc. SPIE, 8446, 84462J

Asada, K., \& Nakamura, M. 2012, ApJ, 745, L28

Atwood, W. B., Abdo, A. A., Ackermann, M., et al. 2009, ApJ, 697, 1071 Banerjee, D. P. K., \& Ashok, N. M. 2012, Bull. Astron. Soc. India, 40, 243

Bentz, M. C., Peterson, B. M., Netzer, H., Pogge, R. W., \& Vestergaard, M. 2009, ApJ, 697, 160

Bessell, M. S., Castelli, F., \& Plez, B. 1998, A\&A, 333, 231

Bonning, E., Urry, C. M., Bailyn, C., et al. 2012, ApJ, 756, 13

Burrows, D. N., Hill, J. E., Nousek, J. A., et al. 2004, Proc. SPIE, 5165, 201

Carrasco, L., Porras, A., Recillas, E., et al. 2015, ATel, 7804

Cawthorne, T. V., Jorstad, S. G., \& Marscher, A. P. 2013, ApJ, 772, 14

Chatterjee, R., Jorstad, S. G., Marscher, A. P., et al. 2008, ApJ, 689, 79

Clarke, D., \& Neumayer, D. 2002, A\&A, 383, 360

Cutri, R. M., Skrutskie, M. F., van Dyk, S., et al. 2003, The IRSA 2MASS All-Sky Point Source Catalog, NASA/IPAC Infrared Science Archive, http://irsa.ipac. caltech.edu/applications/Gator/

D’Arcangelo, F. D., Marscher, A. P., Jorstad, S. G., et al. 2007, ApJ, 659, L107

D'arcangelo, F. D., Marscher, A. P., Jorstad, S. G., et al. 2009, ApJ, 697, 985

de Naurois, M. 2016, ATel, 9102

Domínguez, A., Primack, J. R., Rosario, D. J., et al. 2011, MNRAS, 410, 2556

Dotson, A., Georganopoulos, M., Meyer, E. T., \& McCann, K. 2015, ApJ, 809, 164

Fruck, C., \& Gaug, M. 2015, Eur. Phys. J. Web Conf., 89, 02003

Ghisellini, G., \& Tavecchio, F. 2009, MNRAS, 397, 985

Ghisellini, G., Celotti, A., \& Costamante, L. 2002, A\&A, 386, 833

Ghisellini, G., Tavecchio, F., Foschini, L., et al. 2010, MNRAS, 402, 497

Ghisellini, G., Tavecchio, F., Foschini, L., et al. 2012, MNRAS, 425, 1371

H.E.S.S. Collaboration, Abramowski, A., Acero, F., et al. 2013, A\&A, 554, A107

Hovatta T., Lindfors, E., Blinow, D., et al. 2016, A\&A, 596, A78

Jankowsky, F., Zacharias, M., Wierzcholska, A., et al. 2015, ATel, 7799

Jermak, H., Steele, I. A., Lindfors, E., et al. 2016, MNRAS, 462, 4267

Jorstad, S. G., Marscher, A. P., Lister, M. L., et al. 2005, AJ, 130, 1418

Kalberla, P. M. W., Burton, W. B., Hartmann, D., et al. 2005, A\&A, 440, 775

Larionov, V. M., Jorstad, S. G., Marscher, A. P., et al. 2008, A\&A, 492, 389
MacDonald, N. R., Marscher, A. P., Jorstad, S. G., \& Joshi, M. 2015, ApJ, 804, 111

MAGIC Collaboration, Albert, J., Aliu, E., et al. 2008, Science, 320, 1752

Marscher, A. P., Jorstad, S. G., Larionov, V. M., et al. 2010, ApJ, 710, L126

Mattox J. R., Bertsch, D. L., Chiang, J., et al. 1996, ApJ, 461, 396

Mirzoyan, R. 2016, ATel, 9105

Mirzoyan, R. for the MAGIC Collaboration 2015, ATel, 7542

Poole, T. S., Breeveld, A. A., Page, M. J., et al. 2008, MNRAS, 383, 627

Pushkarev, A. B., Kovalev, Y. Y., Lister, M. L., \& Savolainen, T. 2009, A\&A, 507, L33

Pushkarev, A. B., Hovatta, T., Kovalev, Y. Y., et al. 2012, A\&A, 545, A113

Raiteri, C. M., Villata, M., Bruschini, L., et al. 2010, A\&A, 524, A43

Rolke, W. A., López, A. M., \& Conrad, J. 2005, NIM A, 551, 493

Saito, S., Stawarz, Ł., Tanaka, Y. T., et al. 2013, ApJ, 766, L11

Saito, S., Stawarz, Ł., Tanaka, Y. T., et al. 2015, ApJ, 809, 171

Sameer, Kaur, N., Ganesh, S., Kumar, V., \& Baliyan, K. S. 2015, ATel, 7495

Schlafly, E. F., \& Finkbeiner, D. P. 2011, ApJ, 737, 1038

Sikora, M., Begelman, M. C., \& Rees, M. J. 1994, ApJ, 421, 153

Smith, P. S., Montiel, E., Rightley, S., et al. 2009, 2009 Fermi Symposium, eConf Proceedings C091122 [arXiv: 0912.3621]

Steele, I. A., Smith, R. J., Rees, P. C., et al. 2004, Proc. SPIE, 5489, 679

Tanner, A. M., Bechtold, J., Walker, C. E., Black, J. H., \& Cutri, R. M. 1996, AJ, 112,62

Teraësranta, H., Tornikoski, M., Mujunen, A., et al. 1998, A\&AS, 132, 305

Villata M., Raiteri, C. M., Ghisellini, G., et al. 1997, A\&AS, 121, 119

Zacharias, M., Böttcher, M., Chakraborty, N., et al. 2016, [arXiv: 1611.02098]

Zanin, R., Carmona, E., Sitarek, J., et al., 2013, Proc of 33rd ICRC, Rio de Janeiro, Brazil, 773

Zhang, H., Chen, X., Böttcher, M., Guo, F., \& Li, H. 2015, ApJ, 804, 58

1 ETH Zurich, 8093 Zurich, Switzerland

2 Università di Udine, and INFN Trieste, 33100 Udine, Italy

3 INAF National Institute for Astrophysics, 00136 Rome, Italy

4 Università di Padova and INFN, 35131 Padova, Italy

5 Croatian MAGIC Consortium, Rudjer Boskovic Institute, University of Rijeka, University of Split - FESB, University of Zagreb - FER, University of Osijek, Croatia

6 Saha Institute of Nuclear Physics, 1/AF Bidhannagar, Salt Lake, Sector-1, 700064 Kolkata, India

7 Max-Planck-Institut für Physik, 80805 München, Germany

Universidad Complutense, 28040 Madrid, Spain

9 Inst. de Astrofísica de Canarias, 38200 La Laguna, Tenerife, Spain

10 Universidad de La Laguna, Dpto. Astrofísica, 38206 La Laguna, Tenerife, Spain

11 University of Łódź, 90236 Lodz, Poland

12 Deutsches Elektronen-Synchrotron (DESY), 15738 Zeuthen, Germany

13 Institut de Fisica d'Altes Energies (IFAE), The Barcelona Institute of Science and Technology, Campus UAB, 08193 Bellaterra (Barcelona), Spain

14 Università di Siena, and INFN Pisa, 53100 Siena, Italy

15 Universität Würzburg, 97074 Würzburg, Germany

16 Institute for Space Sciences (CSIC/IEEC), 08193 Barcelona, Spain

17 Technische Universität Dortmund, 44221 Dortmund, Germany

18 Finnish MAGIC Consortium, Tuorla Observatory, University of Turku and Astronomy Division, University of Oulu, Finland

19 Unitat de Física de les Radiacions, Departament de Física, and CERES-IEEC, Universitat Autònoma de Barcelona, 08193 Bellaterra, Spain

20 Universitat de Barcelona, ICC, IEEC-UB, 08028 Barcelona, Spain

21 Japanese MAGIC Consortium, ICRR, The University of Tokyo, Department of Physics and Hakubi Center, Kyoto University, Tokai University, The University of Tokushima, Japan

22 Inst. for Nucl. Research and Nucl. Energy, 1784 Sofia, Bulgaria

23 Università di Pisa, and INFN Pisa, 56126 Pisa, Italy

24 ICREA and Institute for Space Sciences (CSIC/IEEC), 08193 Barcelona, Spain

25 Also at the Department of Physics of Kyoto University, Japan 
${ }^{26}$ Now at Centro Brasileiro de Pesquisas Físicas (CBPF/MCTI), R. Dr. Xavier Sigaud, 150 - Urca, Rio de Janeiro - RJ, 22290-180, Brazil

27 Now at NASA Goddard Space Flight Center, Greenbelt, MD 20771, USA and Department of Physics and Department of Astronomy, University of Maryland, College Park, MD 20742, USA

28 Humboldt University of Berlin, Institut für Physik Newtonstr. 15 12489 Berlin, Germany

29 Also at University of Trieste, Italy

30 Now at École polytechnique fédérale de Lausanne (EPFL), Lausanne, Switzerland

31 Also at Japanese MAGIC Consortium, Japan

32 Now at Finnish Centre for Astronomy with ESO (FINCA), Turku, Finland

33 Also at INAF-Trieste and Dept. of Physics \& Astronomy, University of Bologna, 40126 Bologna, Italy

34 Dip. di Fisica e Astronomia, Università di Bologna, Viale Berti Pichat 6/2, 40127 Bologna, Italy

35 INAF-Istituto di Radioastronomia, via Gobetti 101, 40129 Bologna, Italy

36 KTH Royal Institute of Technology, Department of Physics, AlbaNova, 10691 Stockholm, Sweden

37 Oskar Klein Centre for Cosmoparticle Physics, AlbaNova, 10691 Stockholm, Sweden
38 INAF, Osservatorio Astrofisico di Torino, via Osservatorio 20, 10025 Pino Torinese, Italy

39 Tuorla Observatory, Department of Physics and Astronomy, University of Turku, Finland

40 Aalto University Metsähovi Radio Observatory, Metsähovintie 114, 02540 Kylmälä, Finland

41 Aalto University Department of Radio Science and Engineering, PO Box 13000, 00076 AALTO, Finland

42 IAR, Boston University, 725 Commonwealth Ave, Boston, 02215, USA

43 St. Petersburg State University, Universitetsky prospekt, 28, St. Petersburg 198504, Russia

44 Physical Research Laboratory, Ahmedabad 380009, Gujrat, India

45 Indian Institute of Technology, Gandhinagar 382355, Gujrat, India

46 Department of Astronomy and Astrophysics, The Pennsylvania State University, 532-D, Davey Laboratory, University Park, PA 16802, USA

47 Department of Physics, Lancaster University, Lancaster, LA1 4YW, UK

48 Astrophysics Research Institute, Liverpool John Moores University, Brownlow Hill, Liverpool, L3 5RF, UK

49 Pulkovo Observatory, St. Petersburg, Russia

50 Crimean Astrophysical Observatory, Crimea 